University of Windsor

Scholarship at UWindsor

Winter 2012

\title{
An interval-valued intuitionistic fuzzy multiattribute group decision making framework with incomplete preference over alternatives
}

Zhou-Jing Wang

Kevin W. Li Dr.

University of Windsor

Follow this and additional works at: https://scholar.uwindsor.ca/odettepub

Part of the Business Commons

\section{Recommended Citation}

Wang, Zhou-Jing and Li, Kevin W. Dr.. (2012). An interval-valued intuitionistic fuzzy multiattribute group decision making framework with incomplete preference over alternatives. Expert Systems with Applications, 39 (18), 13509-13516.

https://scholar.uwindsor.ca/odettepub/55

This Article is brought to you for free and open access by the Odette School of Business at Scholarship at UWindsor. It has been accepted for inclusion in Odette School of Business Publications by an authorized administrator of Scholarship at UWindsor. For more information, please contact scholarship@uwindsor.ca. 
1 An interval-valued intuitionistic fuzzy multiattribute group decision making framework

10 Abstract

11 This article proposes a framework to handle multiattribute group decision making 12 problems with incomplete pairwise comparison preference over decision alternatives 13 where qualitative and quantitative attribute values are furnished as linguistic variables 14 and crisp numbers, respectively. Attribute assessments are then converted to interval15 valued intuitionistic fuzzy numbers (IVIFNs) to characterize fuzziness and uncertainty in 16 the evaluation process. Group consistency and inconsistency indices are introduced for 17 incomplete pairwise comparison preference relations on alternatives provided by the 18 decision-makers (DMs). By minimizing the group inconsistency index under certain 19 constraints, an auxiliary linear programming model is developed to obtain unified 20 attribute weights and an interval-valued intuitionistic fuzzy positive ideal solution 21 (IVIFPIS). Attribute weights are subsequently employed to calculate distances between 22 alternatives and the IVIFPIS for ranking alternatives. An illustrative example is provided 23 to demonstrate the applicability and effectiveness of this method.

24 Keywords: Multi-attribute group decision making (MAGDM), interval-valued 25 intuitionistic fuzzy numbers (IVIFNs), linear programming, group consistency and 26 inconsistency

\section{1. Introduction}

28 When facing a decision situation, a decision-maker (DM) often has to evaluate a

\footnotetext{
${ }^{1}$ Corresponding author, Telephone: +1 5192533000 ext. 3456; fax: +1 5199737073.

Email: kwli@uwindsor.ca (K.W. Li), wangzj@xmu.edu.cn (Z.J. Wang).
} 
29 finite set of alternatives against multiple attributes. This process can be conveniently 30 modeled as a multiattribute decision making (MADM) problem. Several formal 31 procedures have been proposed to deal with MADM such as the Technique for Order 32 Preference by Similarity to Ideal Solution (TOPSIS) (Hwang \& Yoon, 1981) and the 33 Linear Programming Technique for Multidimensional Analysis of Preference (LINMAP) 34 (Srinivasan \& Shocker, 1973). The LINMAP proves to be a practical and useful 35 technique for determining attribute weights and a positive-ideal solution based on a DM's 36 pairwise comparisons of alternatives. In the traditional LINMAP, performance ratings are 37 known precisely and given as crisp values. Under many practical decision situations, it is 38 hard, if not impossible, to obtain exact assessment values due to inherent vagueness and 39 uncertainty in human judgment. As such, Zadeh (1965) puts forward a powerful 40 paradigm, fuzzy set theory, to handle ambiguity information that often arises in human 41 decision processes. The LINMAP has subsequently been extended to handle MADM 42 with fuzzy judgment data (Li \& Yang, 2004).

43 In Zadeh's fuzzy set, an element's membership to a particular set is defined as a real 44 value $\mu$ between 0 and 1 and its nonmembership is implied to be $1-\mu$. This extension 45 of traditional binary logic provides a powerful framework to characterize vagueness and 46 uncertainty. The treatment of nonmembership as a complement of membership 47 essentially omits a DM's hesitation in the decision making process. To facilitate further 48 characterization of uncertainty and vagueness, Atanassov (1986) introduces intuitionistic 49 fuzzy sets (IFSs), depicted by real-valued membership, nonmembership, and hesitancy 50 functions. Due to its capability of accommodating hesitation in human decision processes, 51 IFSs have been widely recognized as flexible and practical tools for tackling imprecise 52 and uncertain decision information (Xu \& Cai, 2010) and have been widely applied to the 53 field of decision modeling. For instance, Li (2005) proposes a linear programming 54 method to handle MADM using IFSs; Wei (2010) develops an intuitionistic fuzzy 55 weighted geometric operator-based approach to solve multi-attribute group decision 56 making (MAGDM) problems; Li et al. (2010) extend the LINMAP method to solve 57 MAGDM with intuitionistic fuzzy information.

58 An IFS is characterized by real-valued membership and nonmembership functions 59 defined on $[0,1]$, and the hesitancy function can be easily derived based on the aforesaid 
60 two functions. In some decision situations with highly uncertain and imprecise judgment,

61 it could pose a significant challenge to require that membership and nonmembership be

62 identified as exact values. To address this issue, IFSs are further extended to interval-

63 valued intuitionistic fuzzy sets (IVIFSs) (Atanassov and Gargov, 1989) where

64 membership and nonmembership are represented as interval-valued functions. Since its

65 inception, significant research has been conducted to develop and enrich the IVIFS theory,

66 such as investigations on the correlation and correlation coefficients of IVIFSs (Bustince

67 \& Burillo 1995; Hong, 1998; Hung \& Wu, 2002), fuzzy cross entropy of IVIFSs (Ye,

68 2011), relationships between IFSs, L-fuzzy sets, interval-valued fuzzy sets and IVIFSs

69 (Deschrijver, 2007; Deschrijver, 2008; Deschrijver \& Kerre, 2007), similarity measures

70 of IVIFSs (Wei, Wang, \& Zhang, 2011; Xu \& Chen, 2008), and comparison of the

71 interval-valued intuitionistic numbers (IVIFNs) (Li \& Wang, 2010; Wang, Li, \& Wang,

72 2009; Xu, 2007). Thanks to their advantage in coping with uncertain decision data,

73 IVIFSs have been widely applied to decision models with multiple attributes (Li, 2010a,

74 b; Wang, Li \& Wang, 2009; Park et al., 2011; Li, 2011; Wang, Li, \& Xu, 2011; Wei,

75 2010, 2011; Xu, 2007; Xu \& Yager, 2007, 2008; Xu et al., 2011). Recently, researchers

76 started to address MAGDM problems involving IVIFS decision data. For instance, Park

77 et al. (2009) investigate group decision problems based on correlation coefficients of

78 IVIFSs. Xu (2010) introduces certain IVIFN relations and operations and proposes a

79 distance-based method for group decisions. Ye (2010) develops a MAGDM method with

80 IVIFNs to solve the partner selection problem of a virtual enterprise under incomplete

81 information. Yue (2011) puts forward an approach to aggregate interval numbers into

82 IVIFNs for group decisions. Chen et al. (2011) propose a framework to tackle MAGDM

83 problem based on interval-valued intuitionistic fuzzy preference relations and interval-

84 valued intuitionistic fuzzy decision matrices.

85 To the authors' knowledge, little research has been carried out to handle MAGDM

86 problems in which attribute values are converted to IVIFNs with unknown attribute

87 weights and incomplete pairwise comparison preference relations on alternatives. In this

88 research, the focus is to further extend the LINMAP method and develop a new approach

89 to MAGDM problems with IVIFN decision data. In this paradigm, it is assumed that raw

90 decision data are furnished as linguistic variables (for qualitative attributes) and 
91 numerical values (for quantitative attributes), then IVIFNs are constructed to reflect

92 fuzziness and uncertainty contained in attribute assessment values and DMs' subjective

93 judgment. Group consistency and inconsistency indices are defined for pairwise

94 comparison preference relations on alternatives. A linear program is proposed for

95 deriving the interval-valued intuitionistic fuzzy positive ideal solution (IVIFPIS) and

96 attribute weights. The distances of alternatives to the IVIFPIS are calculated to determine

97 their ranking orders for individual DMs. Finally, a group ranking order can be generated

98 using the Borda function (Hwang \& Yoon, 1981). An earlier version of this paper was

99 presented at a conference and published in the proceedings [Wang, Wang \& Li, 2011].

100 This manuscript has significantly expanded the research reported therein by refining the

101 modeling process, addressing certain technical deficiency, and furnishing two theorems

102 to reveal useful properties of the proposed framework.

103 The remainder of the paper is organized as follows. Section 2 provides preliminaries

104 on IVIFSs and Euclidean distance between IVIFNs. Section 3 formulates the MAGDM

105 problem with IVIFNs and defines group consistency and inconsistency indices. Section 4

106 proposes an approach to handle MAGDM problems with IVIFNs, and a linear program is

107 established to estimate the IVIFPIS and attribute weights. Section 5 presents a numerical

108 example to demonstrate how to apply the proposed approach, followed by some 109 concluding remarks in Section 6.

\section{2. Preliminaries}

111 Let $Z$ be a fixed nonempty universe set, an IFS $A$ in $Z$ is an object in the following 112 form (Atanassov, 1986):

$113 \quad A=\left\{<z, \mu_{A}(z), v_{A}(z)>\mid z \in Z\right\}$,

114 where $\mu_{A}: Z \rightarrow[0,1]$ and $v_{A}: Z \rightarrow[0,1]$, satisfying $0 \leq \mu_{A}(z)+v_{A}(z) \leq 1, \forall z \in Z$.

$115 \mu_{A}(z)$ and $v_{A}(z)$ denote, respectively, the degree of membership and 116 nonmembership of element $z$ to set $A$. In addition, for each IFS $A$ in $Z$, $117 \pi_{A}(z)=1-\mu_{A}(z)-v_{A}(z)$ is often referred to as its intuitionistic fuzzy index, representing 118 the degree of indeterminacy of $z$ to $A$. Obviously, $0 \leq \pi_{A}(z) \leq 1$ for every $z \in Z$.

119 Given that the degrees of membership and nonmembership are sometimes difficult to 120 be derived with exact values, Atanassov and Gargov (1989) extend IFSs to interval- 
121 valued intuitionistic fuzzy sets (IVIFSs) that allow membership and nonmembership 122 functions to assume interval values.

123 Let $D([0,1])$ be the set of all closed subintervals of the unit interval [0,1], an IVIFS

$124 \tilde{A}$ over $Z$ is defined as

$$
\tilde{A}=\left\{<z, \tilde{\mu}_{\tilde{A}}(z), \tilde{v}_{\tilde{A}}(z)>\mid z \in Z\right\},
$$

126 where $\tilde{\mu}_{\tilde{A}}: Z \rightarrow D([0,1]), \tilde{v}_{\tilde{A}}: Z \rightarrow D([0,1])$, and $0 \leq \sup \left(\tilde{\mu}_{\tilde{A}}(z)\right)+\sup \left(\tilde{v}_{\tilde{A}}(z)\right) \leq 1$ for 127 any $z \in Z$.

128 The intervals $\tilde{\mu}_{\tilde{A}}(z)$ and $\tilde{v}_{\tilde{A}}(z)$ define, respectively, the degree of membership and 129 nonmembership of $z$ to $A$. Thus for each $z \in Z$, the difference from an IFS is that $\tilde{\mu}_{\tilde{A}}(z)$ 130 and $\tilde{v}_{\tilde{A}}(z)$ are closed intervals, and their lower and upper bounds are denoted by $131 \tilde{\mu}_{\tilde{A}}^{L}(z), \tilde{\mu}_{\tilde{A}}^{U}(z), \tilde{v}_{\tilde{A}}^{L}(z)$ and $\tilde{v}_{\tilde{A}}^{U}(z)$, respectively. Therefore, the IVIFS $\tilde{A}$ can be 132 equivalently expressed as

$$
\tilde{A}=\left\{<z,\left[\tilde{\mu}_{\tilde{A}}^{L}(z), \tilde{\mu}_{\tilde{A}}^{U}(z)\right],\left[\tilde{v}_{\tilde{A}}^{L}(z), \tilde{v}_{\tilde{A}}^{U}(z)\right]>\mid z \in Z\right\},
$$

134 where $\tilde{\mu}_{\tilde{A}}^{U}(z)+\tilde{v}_{\tilde{A}}^{U}(z) \leq 1,0 \leq \tilde{\mu}_{\tilde{A}}^{L}(z) \leq \tilde{\mu}_{\tilde{A}}^{U}(z) \leq 1,0 \leq \tilde{v}_{\tilde{A}}^{L}(z) \leq \tilde{v}_{\tilde{A}}^{U}(z) \leq 1$.

135 Similar to IFSs, an interval intuitionistic fuzzy index of an element $z \in Z$ is expressed 136 as

$$
\tilde{\pi}_{\tilde{A}}(z)=\left[\tilde{\pi}_{\tilde{A}}^{L}(z), \tilde{\pi}_{\tilde{A}}^{U}(z)\right]=\left[1-\tilde{\mu}_{\tilde{A}}^{U}(z)-\tilde{v}_{\tilde{A}}^{U}(z), 1-\tilde{\mu}_{\tilde{A}}^{L}(z)-\tilde{v}_{\tilde{A}}^{L}(z)\right],
$$

138 which gives the range of hesitancy degree of element $z$ to set $\tilde{A}$.

139 If each of the intervals $\tilde{\mu}_{\tilde{A}}(z)$ and $\tilde{v}_{\tilde{A}}(z)$ contains only a single value, i.e., for every $140 \quad z \in Z, \tilde{\mu}_{\tilde{A}}^{L}(z)=\tilde{\mu}_{\tilde{A}}^{U}(z)$ and $\tilde{v}_{\tilde{A}}^{L}(z)=\tilde{v}_{\tilde{A}}^{U}(z)$, then the given IVIFS $\tilde{A}$ is reduced to a 141 regular IFS.

142 For an IVIFS $\tilde{A}$ and a given $z$, the pair $\left(\tilde{\mu}_{\tilde{A}}(z), \tilde{v}_{\tilde{A}}(z)\right)$ is called an interval-valued 143 intuitionistic fuzzy number (IVIFN) (Wang, Li, \& Wang, 2009; Wang, Li, \& Xu, 2011; $144 \mathrm{Xu}, 2007$; Xu \& Yager, 2008). For convenience, we denote an IVIFN by $([a, b],[c, d])$, 145 where $[a, b] \in D([0,1]),[c, d] \in D([0,1])$ and $b+d \leq 1$.

$146 \mathrm{Xu}$ and Yager (2009) introduce the normalized Hamming distance considering 147 interval intuitionistic fuzzy index between IVIFSs. Here, a normalized Euclidean distance 
148

is introduced to facilitate the discussion in Section 3.

Let $\tilde{\alpha}_{1}=\left(\left[a_{1}, b_{1}\right],\left[c_{1}, d_{1}\right]\right)$ and $\tilde{\alpha}_{2}=\left(\left[a_{2}, b_{2}\right],\left[c_{2}, d_{2}\right]\right)$ be any two IVIFNs, then a normalized Euclidean distance between $\tilde{\alpha}_{1}$ and $\tilde{\alpha}_{2}$ can be defined as:

$$
\begin{aligned}
d\left(\tilde{\alpha}_{1}, \tilde{\alpha}_{2}\right)= & \left(\frac { 1 } { 4 } \left(\left(a_{1}-a_{2}\right)^{2}+\left(b_{1}-b_{2}\right)^{2}+\left(c_{1}-c_{2}\right)^{2}+\left(d_{1}-d_{2}\right)^{2}+\right.\right. \\
& \left.\left.\left(\pi_{\tilde{\alpha}_{1}}^{l}-\pi_{\tilde{\alpha}_{2}}^{l}\right)^{2}+\left(\pi_{\tilde{\alpha}_{1}}^{u}-\pi_{\tilde{\alpha}_{2}}^{u}\right)^{2}\right)\right)^{1 / 2}
\end{aligned}
$$

where $\pi_{\tilde{\alpha}_{1}}^{l}=1-b_{1}-d_{1}, \pi_{\tilde{\alpha}_{1}}^{u}=1-a_{1}-c_{1}, \pi_{\tilde{\alpha}_{2}}^{l}=1-b_{2}-d_{2}, \pi_{\tilde{\alpha}_{2}}^{u}=1-a_{2}-c_{2}$.

\section{An MAGDM problem and group consistency measurement}

This section presents an MAGDM problem with IVIFNs and defines group consistency and inconsistency indices.

\subsection{An MAGDM framework with IVIFN decision data}

Given $n$ feasible decision alternatives $x_{i}(i=1,2, \ldots, n)$ and $m$ qualitative or quantitative attributes $a_{j}(j=1,2, \ldots, m)$. Denote the alternative set by $X=\left\{x_{1}, x_{2}, \ldots, x_{n}\right\}$ and the attribute set by $A=\left\{a_{1}, a_{2}, \ldots, a_{m}\right\}$. The attribute set $A$ can be divided into two mutually exclusive and collectively exhaustive subsets: $A_{1}$ and $A_{2}$, representing the subset of qualitative and quantitative attributes, respectively. It is natural that $A_{1} \cup A_{2}=A$ and $A_{1} \cap A_{2}=\varnothing$, where $\varnothing$ is the empty set. Depending on the decision purpose, an MAGDM problem could be defined as finding the best alternative(s) from all feasible choices or obtaining a ranking for all alternatives based on the information provided by a group of DMs $D=\left\{d_{1}, d_{2}, \ldots, d_{q}\right\}$.

Assume that DM $d_{p} \in D$ assesses each alternative $x_{i} \in X$ on each qualitative attribute $a_{j} \in A_{1}$ as a linguistic variable. These linguistic assessments are then converted into IVIFNs, $\tilde{r}_{i j}^{p}=\left(\left[a_{i j}^{1 p}, b_{i j}^{1 p}\right],\left[c_{i j}^{1 p}, d_{i j}^{1 p}\right]\right)(i=1,2, \ldots, n, p=1,2, \ldots, q)$. The intervals $\left[a_{i j}^{1 p}, b_{i j}^{1 p}\right]$ and $\left[c_{i j}^{1 p}, d_{i j}^{1 p}\right]$ are the degree of satisfaction (or membership) and the degree of nonsatisfaction (or nonmembership) of $x_{i}$ on the qualitative attribute $a_{j}$ with respect to a fuzzy concept "excellence", and satisfy the following conditions: $\left[a_{i j}^{1 p}, b_{i j}^{1 p}\right] \in D([0,1])$, 
Table 1. A conversion table between linguistic variables and IVIFNs

\begin{tabular}{||l|c||}
\hline \multicolumn{1}{|c|}{ Linguistic terms } & IVIFNs \\
\hline Very Good $($ VG) & $([0.90,0.95],[0.02,0.05])$ \\
\hline Good $(\mathrm{G})$ & $([0.70,0.75],[0.20,0.25])$ \\
\hline Fair $(\mathrm{F})$ & $([0.50,0.55],[0.40,0.45])$ \\
\hline Poor $(\mathrm{P})$ & $([0.20,0.25],[0.70,0.75])$ \\
\hline Very Poor $(\mathrm{VP})$ & $([0.02,0.05],[0.90,0.95])$ \\
\hline
\end{tabular}

$172\left[c_{i j}^{1 p}, d_{i j}^{1 p}\right] \in D([0,1])$ and $b_{i j}^{1 p}+d_{i j}^{1 p} \leq 1$. Table 1 furnishes a conversion table between

173 linguistic variables and their corresponding IVIFNs used in the case study in Section 5.

175 For each quantitative attribute $a_{j} \in A_{2}$, it is assumed that each alternative $x_{i} \in X$ is 176 assessed as a numerical value, denoted by $f_{i j}{ }^{p}$. Generally speaking, numerical 177 assessments on different attributes often assume different units (e.g., kilograms for 178 weight and kilometers for distance). In addition, for the same numerical value $f_{i j}^{p}$, 179 different DMs may have different degrees of satisfaction (or membership) and non180 satisfaction (or nonmembership) assessment. As such, it is desirable to convert a 181 numerical value $f_{i j}{ }^{p}$ to dimensionless relative degrees of satisfaction and non-satisfaction, 182 reflecting both objective measurement and $\mathrm{DM} d_{p}$ 's subjective assessment.

183 Quantitative attributes are often classified into two types: benefit and cost attributes. 184 Denote the benefit attribute set by $A_{2}^{b}$ and the cost attribute set by $A_{2}^{c}$. One way to define 185 the relative degree of satisfaction interval $\left[a_{i j}^{2 p}, b_{i j}^{2 p}\right]$ for a numerical value $f_{i j}^{p}$ is given as 186 follows:

$$
\begin{aligned}
& \left\{\begin{array}{l}
a_{i j}^{2 p}=\beta_{j}^{p l}\left(f_{i j}^{p}-f_{j p}^{\min }\right) /\left(f_{j p}^{\max }-f_{j p}^{\min }\right) \\
b_{i j}^{2 p}=\beta_{j}^{p u}\left(f_{i j}^{p}-f_{j p}^{\min }\right) /\left(f_{j p}^{\max }-f_{j p}^{\min }\right)
\end{array} \text { if } a_{j} \in A_{2}^{b},\right. \\
& \left\{\begin{array}{l}
a_{i j}^{2 p}=\beta_{j}^{p l}\left(f_{j p}^{\max }-f_{i j}^{p}\right) /\left(f_{j p}^{\max }-f_{j p}^{\min }\right) \\
b_{i j}^{2 p}=\beta_{j}^{p u}\left(f_{j p}^{\max }-f_{i j}^{p}\right) /\left(f_{j p}^{\max }-f_{j p}^{\min }\right)
\end{array} \text { if } a_{j} \in A_{2}^{c},\right.
\end{aligned}
$$

188 where $f_{j p}^{\max }=\max \left\{f_{i j}{ }^{p} \mid i=1,2, \ldots, n\right\}, f_{j p}^{\min }=\min \left\{f_{i j}{ }^{p} \mid i=1,2, \ldots, n\right\}$ and the parameter $\bar{\beta}_{j}^{p}=\left[\beta_{j}^{p l}, \beta_{j}^{p u}\right] \in D([0,1])$ is given by $\mathrm{DM} d_{p}(p=1,2, \ldots, q)$ according to its expected 190 goals and needs in the decision situation, reflecting the DM's relative degree of 
191 satisfaction (or membership) for the best assessment on attribute $a_{j} \in A_{2}$ (maximum for a

192 benefit attribute or minimum for a cost attribute).

193 It is obvious that $\left[a_{i j}^{2 p}, b_{i j}^{2 p}\right] \in D([0,1])$ and the larger the relative degree interval $194\left[a_{i j}^{2 p}, b_{i j}^{2 p}\right]$, the more satisfying alternative $x_{i}$ is with respect to attribute $a_{j}$.

195 For a numerical value $f_{i j}^{p}\left(i=1,2, \ldots, n, a_{j} \in A_{2}\right)$, let

$$
f_{i j}^{\prime p}=\kappa_{j}^{p} f_{i j}^{p}+\lambda_{j}^{p}
$$

197 where $\kappa_{j}^{p}>0$ and $\lambda_{j}^{p}$ are constants given by the $\mathrm{DM} d_{p}(p=1,2, \ldots, q)$. The purpose of 198 introducing this linear transformation formula is to accommodate the case that DM $d_{p}$ 199 may adopt a different rating system for a quantitative attribute $a_{j} \in A_{2}$. Next, Theorem 3.1 200 establishes that the relative degree of satisfaction interval for a numerical value $f_{i j}^{p}$ 201 remains the same for its converted value $f_{i j}^{\prime p}$ under the transformation relation (3.2).

202 Theorem 3.1 For a numerical assessment $f_{i j}^{p}$ and its converted value $f_{i j}^{{ }^{\prime} p}$ based on Eq.

203 (3.2), denote their relative degree of satisfaction intervals by $\left[a_{i j}^{2 p}, b_{i j}^{2 p}\right]$ and $\left[a_{i j}^{\prime 2 p}, b_{i j}^{\prime 2 p}\right]$,

204 then $a_{i j}^{2 p}=a_{i j}^{\prime 2 p}$ and $b_{i j}^{2 p}=b_{i j}^{\prime 2 p}$.

205 Proof. Since

206

$$
\begin{aligned}
f_{j p}^{\prime \max } & =\max \left\{\kappa_{j}^{p} f_{i j}^{p}+\lambda_{j}^{p} \mid i=1,2, \ldots, n\right\} \\
& =\kappa_{j}^{p} \max \left\{f_{i j}^{p} \mid i=1,2, \ldots, n\right\}+\lambda_{j}^{p} \\
& =\kappa_{j}^{p} f_{j p}^{\max }+\lambda_{j}^{p}
\end{aligned}
$$

207 and

208

$$
\begin{aligned}
f_{j p}^{\prime \min } & =\min \left\{\kappa_{j}^{p} f_{i j}^{p}+\lambda_{j}^{p} \mid i=1,2, \ldots, n\right\} \\
& =\kappa_{j}^{p} \min \left\{f_{i j}^{p} \mid i=1,2, \ldots, n\right\}+\lambda_{j}^{p} \\
& =\kappa_{j}^{p} f_{j p}^{\min }+\lambda_{j}^{p}
\end{aligned}
$$

209 Then, 


$$
\begin{aligned}
& \left(a_{i j}^{\prime 2 p}=\beta_{j}^{p l}\left(f_{i j}^{\prime p}-f_{j p}^{\prime \min }\right) /\left(f_{j p}^{\prime \max }-f_{j p}^{\prime \min }\right)\right. \\
& =\beta_{j}^{p l}\left(\kappa_{j}^{p} f_{i j}^{p}+\lambda_{j}^{p}-\left(\kappa_{j}^{p} f_{j p}^{\min }+\lambda_{j}^{p}\right)\right) /\left(\left(\kappa_{j}^{p} f_{j p}^{\max }+\lambda_{j}^{p}\right)-\left(\kappa_{j}^{p} f_{j p}^{\min }+\lambda_{j}^{p}\right)\right) \\
& =\beta_{j}^{p l}\left(f_{i j}^{p}-f_{j p}^{\min }\right) /\left(f_{j p}^{\max }-f_{j p}^{\min }\right)=a_{i j}^{2 p} \\
& b_{i j}^{\prime 2 p}=\beta_{j}^{p u}\left(f_{i j}^{\prime p}-f_{j p}^{\min }\right) /\left(f_{j p}^{\prime \max }-f_{j p}^{\prime \min }\right) \\
& =\beta_{j}^{p u}\left(\kappa_{j}^{p} f_{i j}^{p}+\lambda_{j}^{p}-\left(\kappa_{j}^{p} f_{j p}^{\min }+\lambda_{j}^{p}\right)\right) /\left(\left(\kappa_{j}^{p} f_{j p}^{\max }+\lambda_{j}^{p}\right)-\left(\kappa_{j}^{p} f_{j p}^{\min }+\lambda_{j}^{p}\right)\right) \\
& =\beta_{j}^{p u}\left(f_{i j}^{p}-f_{j p}^{\min }\right) /\left(f_{j p}^{\max }-f_{j p}^{\min }\right)=b_{i j}^{2 p} \\
& \left(a_{i j}^{\prime 2 p}=\beta_{j}^{p l}\left(f_{j p}^{\prime \max }-f_{i j}^{\prime p}\right) /\left(f_{j p}^{\prime \max }-f_{j p}^{\prime \min }\right)\right. \\
& =\beta_{j}^{p l}\left(\kappa_{j}^{p} f_{j p}^{\max }+\lambda_{j}^{p}-\left(\kappa_{j}^{p} f_{i j}^{p}+\lambda_{j}^{p}\right)\right) /\left(\left(\kappa_{j}^{p} f_{j p}^{\max }+\lambda_{j}^{p}\right)-\left(\kappa_{j}^{p} f_{j p}^{\min }+\lambda_{j}^{p}\right)\right) \\
& =\beta_{j}^{p l}\left(f_{j p}^{\max }-f_{i j}^{p}\right) /\left(f_{j p}^{\max }-f_{j p}^{\min }\right)=a_{i j}^{2 p} \\
& b_{i j}^{\prime 2 p}=\beta_{j}^{p u}\left(f_{j p}^{\prime \max }-f_{i j}^{\prime p}\right) /\left(f_{j p}^{\prime \max }-f_{j p}^{\prime \min }\right) \\
& =\beta_{j}^{p u}\left(\kappa_{j}^{p} f_{j p}^{\max }+\lambda_{j}^{p}-\left(\kappa_{j}^{p} f_{i j}^{p}+\lambda_{j}^{p}\right)\right) /\left(\left(\kappa_{j}^{p} f_{j p}^{\max }+\lambda_{j}^{p}\right)-\left(\kappa_{j}^{p} f_{j p}^{\min }+\lambda_{j}^{p}\right)\right) \\
& =\beta_{j}^{p u}\left(f_{j p}^{\max }-f_{i j}^{p}\right) /\left(f_{j p}^{\max }-f_{j p}^{\min }\right)=b_{i j}^{2 p}
\end{aligned}
$$

212 The proof of Theorem 3.1 is thus completed.

213 Theorem 3.1 guarantees that Eq. (3.1) always yields the same relative degree of 214 satisfaction interval for a numerical assessment even if it is converted to a different rating 215 system as long as the conversion process follows the linear relationship in Eq. (3.2).

216 Similarly, assume that $\mathrm{DM} d_{p}(p=1,2, \ldots, q)$ gives its relative degree of non217 satisfaction interval as $\left[\hat{c}_{j}^{2 p}, \hat{d}_{j}^{2 p}\right]$ for the best assessment on attribute $a_{j} \in A_{2}$ (maximum

218 value $f_{j p}^{\max }$ for a benefit attribute or minimum value $f_{j p}^{\min }$ for a cost attribute), where $\hat{d}_{j}^{2 p}+\beta_{j}^{p u} \leq 1$ for all $a_{j} \in A_{2}$.

220

Let

$$
\gamma_{j}^{p l}=\left\{\begin{array}{cc}
\frac{\tilde{c}_{j}^{2 p}}{1-\beta_{j}^{p u}} & \beta_{j}^{p u}<1 \\
0 & \beta_{j}^{p u}=1
\end{array}\right.
$$

$$
\gamma_{j}^{p u}=\left\{\begin{array}{cc}
\frac{\tilde{d}_{j}^{2 p}}{1-\beta_{j}^{p u}} & \beta_{j}^{p u}<1 \\
0 & \beta_{j}^{p u}=1
\end{array}\right.
$$


222 Obviously, $\gamma_{j}^{p l} \leq \gamma_{j}^{p u}$ and $\left[\gamma_{j}^{p l}, \gamma_{j}^{p u}\right] \in D([0,1])$. Denote $\bar{\gamma}_{j}^{p} \triangleq\left[\gamma_{j}^{p l}, \gamma_{j}^{p u}\right]$, then DM $d_{p}$ 's

223 relative degree of non-satisfaction interval $\left[c_{i j}^{2 p}, d_{i j}^{2 p}\right]$ for the numerical value $f_{i j}^{p}$ can be

224 computed by the following formula:

$$
\left[c_{i j}^{2 p}, d_{i j}^{2 p}\right]=\left(1-b_{i j}^{2 p}\right) \bar{\gamma}_{j}^{p}=\left[\gamma_{j}^{p l}\left(1-b_{i j}^{2 p}\right), \gamma_{j}^{p u}\left(1-b_{i j}^{2 p}\right)\right]
$$

As $0 \leq \gamma_{j}^{p u} \leq 1$ and $0 \leq b_{i j}^{2 p} \leq 1$, it follows that $0 \leq b_{i j}^{2 p}+\gamma_{j}^{p u}\left(1-b_{i j}^{2 p}\right) \leq b_{i j}^{2 p}+1-b_{i j}^{2 p}=1$, we

227 have $0 \leq b_{i j}^{2 p}+d_{i j}^{2 p} \leq 1$. Therefore, Eqs. (3.1) and (3.4) ensure that a numerical assessment

$228 f_{i j}^{p}$ is transformed into an IVIFN, $\left(\left[a_{i j}^{2 p}, b_{i j}^{2 p}\right],\left[c_{i j}^{2 p}, d_{i j}^{2 p}\right]\right)$.

$229 \quad$ Let

$$
\tilde{r}_{i j}^{p}=\left(\left[a_{i j}^{p}, b_{i j}^{p}\right],\left[c_{i j}^{p}, d_{i j}^{p}\right]\right)= \begin{cases}\left(\left[a_{i j}^{1 p}, b_{i j}^{1 p}\right],\left[c_{i j}^{1 p}, d_{i j}^{1 p}\right]\right) & \text { if } a_{j} \in A_{1} \\ \left(\left[a_{i j}^{2 p}, b_{i j}^{2 p}\right],\left[c_{i j}^{2 p}, d_{i j}^{2 p}\right]\right) & \text { if } a_{j} \in A_{2}\end{cases}
$$

231 where $i=1,2, \ldots, n$ and $j=1,2, \ldots, m$. Thus, an MAGDM problem with IVIFNs can be 232 concisely expressed in an IVIFN matrix format as follows:

$$
\tilde{R}^{p}=\left(\tilde{r}_{i j}^{p}\right)_{n \times m}=\left(\left(\left[a_{i j}^{p}, b_{i j}^{p}\right],\left[c_{i j}^{p}, d_{i j}^{p}\right]\right)\right)_{n \times m}, \quad(p=1,2, \ldots, q)
$$

\subsection{Group consistency and inconsistency}

236 In an MAGDM problem, different attribute weights reflect their varying importance in selecting the final alternative. Let $\omega=\left(\omega_{1}, \omega_{2}, \ldots, \omega_{m}\right)^{T}$ be the unknown attribute weight vector, where $\omega_{j} \geq 0, j=1,2, \ldots, m$, and the weights are often normalized to one, i.e.

$239 \sum_{j=1}^{m} \omega_{j}=1$. Denote the unknown interval-valued intuitionistic fuzzy positive ideal 240 solution (IVIFPIS) by $x^{*}=\left(\tilde{r}_{1}^{*}, \tilde{r}_{2}^{*}, \ldots, \tilde{r}_{m}^{*}\right)^{T}$, where $\tilde{r}_{j}^{*}=\left(\left[a_{j}^{*}, b_{j}^{*}\right],\left[c_{j}^{*}, d_{j}^{*}\right]\right)(j=1,2, \ldots, m)$

241 are IVIFNs. Then the weighted average of squared Euclidean distance between DM $d_{p}$ 's 242 assessment vector $x_{i}^{p}=\left(\tilde{r}_{i 1}^{p}, \tilde{r}_{i 2}^{p}, \ldots, \tilde{r}_{i m}^{p}\right)$ and the IVIFPIS $x^{*}=\left(\tilde{r}_{1}^{*}, \tilde{r}_{2}^{*}, \ldots, \tilde{r}_{m}^{*}\right)^{T}$ can be 243 defined as follows:

$$
S_{i}^{p}=\sum_{j=1}^{m} \omega_{j}\left[d\left(\tilde{r}_{i j}^{p}, \tilde{r}_{j}^{*}\right)\right]^{2}
$$

245 By (2.1), $S_{i}^{p}$ can be expanded as: 


$$
\begin{gathered}
S_{i}^{p}=\frac{1}{4} \sum_{j=1}^{m} \omega_{j}\left[\left(a_{i j}^{p}-a_{j}^{*}\right)^{2}+\left(b_{i j}^{p}-b_{j}^{*}\right)^{2}+\left(c_{i j}^{p}-c_{j}^{*}\right)^{2}+\left(d_{i j}^{p}-d_{j}^{*}\right)^{2}+\right. \\
\left.\left(\pi_{i j}^{p l}-\pi_{j}^{* l}\right)^{2}+\left(\pi_{i j}^{p u}-\pi_{j}^{* u}\right)^{2}\right]
\end{gathered}
$$

where $\pi_{i j}^{p l}=1-b_{i j}^{p}-d_{i j}^{p}, \pi_{i j}^{p u}=1-a_{i j}^{p}-c_{i j}^{p}, \pi_{j}^{* l}=1-b_{j}^{*}-d_{j}^{*}$ and $\pi_{j}^{* u}=1-a_{j}^{*}-c_{j}^{*}$.

Let

$$
\begin{aligned}
& F_{i j}^{p}=\frac{1}{4}\left[\left(a_{i j}^{p}\right)^{2}+\left(b_{i j}^{p}\right)^{2}+\left(c_{i j}^{p}\right)^{2}+\left(d_{i j}^{p}\right)^{2}+\left(\pi_{i j}^{p l}\right)^{2}+\left(\pi_{i j}^{p u}\right)^{2}-2 \pi_{i j}^{p l}-2 \pi_{i j}^{p u}\right], \\
& C_{i j}^{p}=\frac{1}{2}\left(-a_{i j}^{p}+\pi_{i j}^{p u}\right), G_{i j}^{p}=\frac{1}{2}\left(-b_{i j}^{p}+\pi_{i j}^{p l}\right), \\
& H_{i j}^{p}=\frac{1}{2}\left(-C_{i j}^{p}+\pi_{i j}^{p u}\right), T_{i j}^{p}=\frac{1}{2}\left(-d_{i j}^{p}+\pi_{i j}^{p l}\right)
\end{aligned}
$$

250 and

$$
\hat{a}_{j}=\omega_{j} a_{j}^{*}, \hat{b}_{j}=\omega_{j} b_{j}^{*}, \hat{c}_{j}=\omega_{j} c_{j}^{*}, \hat{d}_{j}=\omega_{j} d_{j}^{*}
$$

252 for each $i=1,2, \ldots, n, j=1,2, \ldots, m$. Then $S_{i}^{p}$ can be written as:

$$
\begin{array}{r}
S_{i}^{p}=\sum_{j=1}^{m} \omega_{j} F_{i j}^{p}+\sum_{j=1}^{m} \hat{a}_{j} C_{i j}^{p}+\sum_{j=1}^{m} \hat{b}_{j} G_{i j}^{p}+\sum_{j=1}^{m} \hat{c}_{j} H_{i j}^{p}+\sum_{j=1}^{m} \hat{d}_{j} T_{i j}^{p}+ \\
\frac{1}{4} \sum_{j=1}^{m} \omega_{j}\left[\left(a_{j}^{*}\right)^{2}+\left(b_{j}^{*}\right)^{2}+\left(c_{j}^{*}\right)^{2}+\left(d_{j}^{*}\right)^{2}+\left(\pi_{j}^{* l}\right)^{2}+\left(\pi_{j}^{* u}\right)^{2}\right]
\end{array}
$$

254 If the weight vector $\omega$ and the IVIFPIS $x^{*}$ are given by the DMs, then $S_{i}^{p}(i=1$, $2552, \ldots, n)$ can be calculated by using (3.11). A ranking of alternatives can thus be 256 conveniently obtained for $\mathrm{DM} d_{p}$ based on $S_{i}^{p}$. However, in this paper, it is conceived 257 that the weight vector $\omega$ and the IVIFPIS $x^{*}$ are not provided by the DMs. Instead, based 258 on incomplete pairwise comparisons of alternatives, a model is proposed to generate a 259 best compromise alternative as the solution that has the shortest distance to the IVIFPIS. 260 To accomplish this goal, consistency and inconsistency indices are introduced based on $261 S_{i}^{p}$ and incomplete pairwise preference relations on alternatives furnished by the DMs.

262 Assume that $\mathrm{DM} d_{p} \in D(p=1,2, \ldots, q)$ provides its comparison preference relations 263 on alternatives as $\Omega^{p}=\left\{(k, t) \mid x_{k} \succsim_{p} x_{t}, k, t \in\{1,2, \ldots, n\}\right\}$, where $x_{k} \succsim_{p} x_{t}$ indicates that $264 \mathrm{DM} d_{p}$ prefers $x_{k}$ to $x_{t}$ or is indifferent between $x_{k}$ and $x_{t}$. 
By (3.7), $S_{t}^{p} \geq S_{k}^{p}$ means that alternative $x_{k}$ is closer to the IVIFPIS $x^{*}$ compared to 266 alternative $x_{t}$. In this case, the ranking order of alternatives $x_{k}$ and $x_{t}$ implied by the 267 normalized Euclidean distance is $x_{k} \succsim_{p} x_{t}$. If $\mathrm{DM} d_{p}$ furnishes the same pairwise 268 comparison result for these two alternatives, i.e., $(k, t) \in \Omega^{p}$, the ranking is called 269 consistent. Otherwise, if the computed distance reveals $S_{t}^{p}<S_{k}^{p}$, but the ranking order 270 furnished by the DM is $x_{k} \succsim_{p} x_{t}$, this ranking is referred to as inconsistent. This 271 inconsistency indicates that the weights and IVIFPIS $x^{*}$ are not chosen properly. Next, 272 the consistency index of DM $d_{p}$ is introduced as follows:

$$
E^{p}=\sum_{(k, t) \in \Omega^{p}} \max \left\{0, S_{t}^{p}-S_{k}^{p}\right\}
$$

274 and the group consistency index is thus calculated as:

275

$$
E=\sum_{p=1}^{q} E^{p}=\sum_{p=1}^{q} \sum_{(k, t) \in \Omega^{p}} \max \left\{0, S_{t}^{p}-S_{k}^{p}\right\}
$$

Similarly, the inconsistency index of DM $d_{p}$ is defined as:

$$
B^{p}=\sum_{(k, t) \in \Omega^{p}} \max \left\{0, S_{k}^{p}-S_{t}^{p}\right\}
$$

278 and the group inconsistency index is determined as:

$$
B=\sum_{p=1}^{q} B^{p}=\sum_{p=1}^{q} \sum_{(k, t) \in \Omega^{p}} \max \left\{0, S_{k}^{p}-S_{t}^{p}\right\}
$$

280

Let

282 for each $i, s=1,2, \ldots, n, j=1,2, \ldots, m$. Then it follows from (3.11) that

$$
F_{i j s}^{p}=F_{i j}^{p}-F_{s j}^{p}, C_{i j s}^{p}=C_{i j}^{p}-C_{s j}^{p}, G_{i j s}^{p}=G_{i j}^{p}-G_{s j}^{p}, H_{i j s}^{p}=H_{i j}^{p}-H_{s j}^{p}, T_{i j s}^{p}=T_{i j}^{p}-T_{s j}^{p}
$$

283

$$
\begin{aligned}
& \max \left\{0, S_{i}^{p}-S_{s}^{p}\right\}-\max \left\{0, S_{s}^{p}-S_{i}^{p}\right\}=S_{i}^{p}-S_{s}^{p} \\
& =\sum_{j=1}^{m} \omega_{j} F_{i j s}^{p}+\sum_{j=1}^{m} \hat{a}_{j} C_{i j s}^{p}+\sum_{j=1}^{m} \hat{b}_{j} G_{i j s}^{p}+\sum_{j=1}^{m} \hat{c}_{j} H_{i j s}^{p}+\sum_{j=1}^{m} \hat{d}_{j} T_{i j s}^{p}
\end{aligned}
$$

284 for each $i, s=1,2, \ldots, n$. From (3.13), (3.15) and (3.17), one can obtain that 


$$
\begin{aligned}
E-B= & \sum_{p=1}^{q} \sum_{(k, t) \in \Omega^{p}}\left(\max \left\{0, S_{t}^{p}-S_{k}^{p}\right\}-\max \left\{0, S_{k}^{p}-S_{t}^{p}\right\}\right) \\
= & \sum_{p=1}^{q} \sum_{(k, t) \in \Omega^{p}}\left(S_{t}^{p}-S_{k}^{p}\right) \\
= & \sum_{j=1}^{m} \omega_{j}\left(\sum_{p=1}^{q} \sum_{(k, t) \in \Omega^{p}} F_{t j k}^{p}\right)+\sum_{j=1}^{m} \hat{a}_{j}\left(\sum_{p=1}^{q} \sum_{(k, t) \in \Omega^{p}} C_{t j k}^{p}\right)+\sum_{j=1}^{m} \hat{b}_{j}\left(\sum_{p=1}^{q} \sum_{(k, t) \in \Omega^{p}} G_{t j k}^{p}\right)+ \\
& \sum_{j=1}^{m} \hat{c}_{j}\left(\sum_{p=1}^{q} \sum_{(k, t) \in \Omega^{p}} H_{t j k}^{p}\right)+\sum_{j=1}^{m} \hat{d}_{j}\left(\sum_{p=1}^{q} \sum_{(k, t) \in \Omega^{p}} T_{t j k}^{p}\right) .
\end{aligned}
$$

Denote

$$
F_{j}=\sum_{p=1}^{q} \sum_{(k, t) \in \Omega^{p}} F_{t j k}^{p}, C_{j}=\sum_{p=1}^{q} \sum_{(k, t) \in \Omega^{p}} C_{t j k}^{p}, G_{j}=\sum_{p=1}^{q} \sum_{(k, t) \in \Omega^{p}} G_{t j k}^{p}, H_{j}=\sum_{p=1}^{q} \sum_{(k, t) \in \Omega^{p}} H_{t j k}^{p}, T_{j}=\sum_{p=1}^{q} \sum_{(k, t) \in \Omega^{p}} T_{t j k}^{p}
$$

Then, Eq. (3.18) can be simply rewritten as follows:

$$
E-B=\sum_{j=1}^{m} \omega_{j} F_{j}+\sum_{j=1}^{m} \hat{a}_{j} C_{j}+\sum_{j=1}^{m} \hat{b}_{j} G_{j}+\sum_{j=1}^{m} \hat{c}_{j} H_{j}+\sum_{j=1}^{m} \hat{d}_{j} T_{j}
$$

\section{A linear programming approach to the MAGDM problem}

As the group inconsistency index $B$ reflects the overall inconsistency between the derived Euclidean distance and the DMs' judgment, the smaller the $B$, the better the model characterizes the DMs' decision rationales. Therefore, a sensible attribute weight vector $\omega=\left(\omega_{1}, \omega_{2}, \ldots, \omega_{m}\right)^{T}$ and IVIFPIS $x^{*}$ is to minimize the group inconsistency index $B$ (Li et al. (2010) apply the similar treatment to handle multiattribute group decision making with intuitionistic fuzzy sets). Based on this consideration, the following optimization model is established to determine $\omega$ and $x^{*}$ :

$$
\begin{array}{ll}
\min & \{B\} \\
\text { s.t. } & E-B \geq h \\
& b_{j}^{*}+d_{j}^{*} \leq 1, a_{j}^{*} \leq b_{j}^{*}, c_{j}^{*} \leq d_{j}^{*} \quad(j=1,2, \ldots, m) \\
& a_{j}^{*} \geq 0, b_{j}^{*} \geq 0, c_{j}^{*} \geq 0, d_{j}^{*} \geq 0 \quad(j=1,2, \ldots, m) \\
& \omega_{j} \geq 0 \quad(j=1,2, \ldots, m) .
\end{array}
$$

where $h$ is a positive number that is expected to reflect by how much the consistency index should exceed the inconsistency index for the group of DMs. 


$$
\begin{aligned}
& \min \left\{\sum_{p=1}^{q} \sum_{(k, t) \in \Omega^{p}} \max \left\{0, S_{k}^{p}-S_{t}^{p}\right\}\right\} \\
& \text { s.t } \sum_{j=1}^{m} \omega_{j} F_{j}+\sum_{j=1}^{m} \hat{a}_{j} C_{j}+\sum_{j=1}^{m} \hat{b}_{j} G_{j}+\sum_{j=1}^{m} \hat{c}_{j} H_{j}+\sum_{j=1}^{m} \hat{d}_{j} T_{j} \geq h \\
& b_{j}^{*}+d_{j}^{*} \leq 1, a_{j}^{*} \leq b_{j}^{*}, c_{j}^{*} \leq d_{j}^{*} \quad(j=1,2, \ldots, m) \\
& a_{j}^{*} \geq 0, b_{j}^{*} \geq 0, c_{j}^{*} \geq 0, d_{j}^{*} \geq 0 \quad(j=1,2, \ldots, m) \\
& \omega_{j} \geq 0 \quad(j=1,2, \ldots, m) .
\end{aligned}
$$

304 For each pair of alternatives $(k, t) \in \Omega^{p}$, let $\xi_{k t}^{p} \triangleq \max \left(0, S_{k}^{p}-S_{t}^{p}\right)$, then

$305 \xi_{k t}^{p} \geq-\left(S_{t}^{p}-S_{k}^{p}\right)$, i.e., $\left(S_{t}^{p}-S_{k}^{p}\right)+\xi_{k t}^{p} \geq 0$. It follows from (3.17) that

306

$$
\sum_{j=1}^{m} \omega_{j} F_{t j k}^{p}+\sum_{j=1}^{m} \hat{a}_{j} C_{t j k}^{p}+\sum_{j=1}^{m} \hat{b}_{j} G_{t j k}^{p}+\sum_{j=1}^{m} \hat{c}_{j} H_{t j k}^{p}+\sum_{j=1}^{m} \hat{d}_{j} T_{t j k}^{p}+\xi_{k t}^{p} \geq 0
$$

As $\hat{a}_{j}=\omega_{j} a_{j}^{*}, \hat{b}_{j}=\omega_{j} b_{j}^{*}, \hat{c}_{j}=\omega_{j} c_{j}^{*}, \hat{d}_{j}=\omega_{j} d_{j}^{*}(j=1,2, \ldots, m)$, one can confirm

308 that $\hat{a}_{j} \leq \hat{b}_{j}, \hat{c}_{j} \leq \hat{d}_{j}$ since $a_{j}^{*} \leq b_{j}^{*}, c_{j}^{*} \leq d_{j}^{*}$, and $\hat{b}_{j}+\hat{d}_{j} \leq \omega_{j}$ due to $b_{j}^{*}+d_{j}^{*} \leq 1$ for $j=1$,

$3092, \ldots, m$. By incorporating (4.3) as a constraint, the nonlinear model (4.2) is transformed 310 to the following linear program by treating $\xi_{k t}^{p}$ as free decision variables:

$$
\begin{aligned}
& \min \left\{\sum_{p=1}^{q} \sum_{(k, t) \in \Omega^{p}} \xi_{k t}^{p}\right\} \\
& \text { s.t. } \sum_{j=1}^{m} \omega_{j} F_{j}+\sum_{j=1}^{m} \hat{a}_{j} C_{j}+\sum_{j=1}^{m} \hat{b}_{j} G_{j}+\sum_{j=1}^{m} \hat{c}_{j} H_{j}+\sum_{j=1}^{m} \hat{d}_{j} T_{j} \geq h \\
& \quad \sum_{j=1}^{m} \omega_{j} F_{t j k}^{p}+\sum_{j=1}^{m} \hat{a}_{j} C_{t j k}^{p}+\sum_{j=1}^{m} \hat{b}_{j} G_{t j k}^{p}+\sum_{j=1}^{m} \hat{c}_{j} H_{t j k}^{p}+\sum_{j=1}^{m} \hat{d}_{j} T_{t j k}^{p}+\xi_{k t}^{p} \geq 0 \\
& \quad\left((k, t) \in \Omega^{p} ; p=1,2, \ldots, q\right) \\
& \xi_{k t}^{p} \geq 0 \quad\left((k, t) \in \Omega^{p} ; p=1,2, \ldots, q\right) \\
& \hat{b}_{j}+\hat{d}_{j} \leq \omega_{j}, \hat{a}_{j} \leq \hat{b}_{j}, \hat{c}_{j} \leq \hat{d}_{j} \quad(j=1,2, \ldots, m) \\
& \hat{a}_{j} \geq 0, \hat{b}_{j} \geq 0, \hat{c}_{j} \geq 0, \hat{d}_{j} \geq 0 \quad(j=1,2, \ldots, m) \\
& \quad \omega_{j} \geq 0 \quad(j=1,2, \ldots, m) .
\end{aligned}
$$

312 It is apparent that the optimal solution of (4.4) depends on the parameter $h$. Denote 313 the optimal solution by $\left(\omega_{1}^{0}(h), \omega_{2}^{0}(h), \ldots, \omega_{m}^{0}(h)\right), \quad\left(\hat{a}_{1}^{0}(h), \hat{a}_{2}^{0}(h), \ldots, \hat{a}_{m}^{0}(h)\right)$ $314\left(\hat{b}_{1}^{0}(h), \hat{b}_{2}^{0}(h), \ldots, \hat{b}_{m}^{0}(h)\right), \quad\left(\hat{c}_{1}^{0}(h), \hat{c}_{2}^{0}(h), \ldots, \hat{c}_{m}^{0}(h)\right), \quad\left(\hat{d}_{1}^{0}(h), \hat{d}_{2}^{0}(h), \ldots, \hat{d}_{m}^{0}(h)\right) \quad, \quad$ and 
$\left(\left(\xi_{k t}^{0 p}(h)\right)_{(k, t) \in \Omega^{p}}\right)(p=1,2, \ldots, q)$, respectively.

316 Given the constraints $\hat{b}_{j}+\hat{d}_{j} \leq \omega_{j}, \hat{a}_{j} \leq \hat{b}_{j}, \hat{c}_{j} \leq \hat{d}_{j}, \hat{a}_{j} \geq 0, \hat{b}_{j} \geq 0, \hat{c}_{j} \geq 0, \hat{d}_{j} \geq 0$

$317(j=1,2, \ldots, m)$ in (4.4), it follows that $\hat{a}_{j}=0, \hat{b}_{j}=0, \hat{c}_{j}=0, \hat{d}_{j}=0$ if $\omega_{j}=0$, and $318 \frac{\hat{b}_{j}}{\omega_{j}}+\frac{\hat{d}_{j}}{\omega_{j}} \leq 1$ if $\omega_{j}>0$. Therefore, the optimal values of $a_{j}^{*}, b_{j}^{*} c_{j}^{*}, d_{j}^{*}(j=1,2, \ldots, m)$,

319 denoted by $a_{j}^{* 0}(h), b_{j}^{* 0}(h), c_{j}^{*_{0}}(h), d_{j}^{* 0}(h)$, can be computed using (3.10) as follows:

320

$$
a_{j}^{* 0}(h)=\left\{\begin{array}{cl}
\frac{\hat{a}_{j}^{0}(h)}{\omega_{j}^{0}(h)} & \text { if } \omega_{j}^{0}(h)>0 \\
0 & \text { if } \omega_{j}^{0}(h)=0
\end{array}, b_{j}^{* 0}(h)=\left\{\begin{array}{cc}
\frac{\hat{b}_{j}^{0}(h)}{\omega_{j}^{0}(h)} & \text { if } \omega_{j}^{0}(h)>0 \\
0 & \text { if } \omega_{j}^{0}(h)=0
\end{array},\right.\right.
$$

$$
c_{j}^{* 0}(h)=\left\{\begin{array}{cl}
\frac{\hat{c}_{j}^{0}(h)}{\omega_{j}^{0}(h)} & \text { if } \omega_{j}^{0}(h)>0 \\
0 & \text { if } \omega_{j}^{0}(h)=0
\end{array}, d_{j}^{* 0}(h)=\left\{\begin{array}{cc}
\frac{\hat{d}_{j}^{0}(h)}{\omega_{j}^{0}(h)} & \text { if } \omega_{j}^{0}(h)>0 \\
0 & \text { if } \omega_{j}^{0}(h)=0
\end{array}\right.\right.
$$

321 It is clear that $\omega_{j}^{0}(h)=0$ corresponds to the case that attribute $a_{j}$ does not contribute 322 to the distance $S_{i}^{p}$ between alternative $x_{i}$ and the IVIFPIS. In this case, $a_{j}$ is irrelevant 323 in determining $\mathrm{DM} d_{p}$ 's preference.

324 It is easy to verify that $\left[a_{j}^{* 0}(h), b_{j}^{* 0}(h)\right] \in D([0,1]),\left[c_{j}^{* 0}(h), d_{j}^{* 0}(h)\right] \in D([0,1])$ and 325 $b_{j}^{* 0}(h)+d_{j}^{*_{0}}(h) \leq 1$. Let $\tilde{r}_{j}^{* 0}(h)=\left(\left[a_{j}^{* 0}(h), b_{j}^{*_{0}}(h)\right],\left[c_{j}^{*_{0}}(h), d_{j}^{* 0}(h)\right]\right)(j=1,2, \ldots, m)$. Thus, an optimal IVIFPIS, denoted by $x^{* 0}(h)=\left(\tilde{r}_{1}^{* 0}(h), \tilde{r}_{2}^{* 0}(h), \ldots, \tilde{r}_{m}^{* 0}(h)\right)^{T}$, is determined.

As linear program (4.4) does not include a weight normalization condition, the optimal weight vector $\left(\omega_{1}^{0}(h), \omega_{2}^{0}(h), \ldots, \omega_{m}^{0}(h)\right)^{T}$ should then be normalized as

$$
\left(\omega_{1}^{0}(h) / \sum_{j=1}^{m} \omega_{j}^{0}(h), \omega_{2}^{0}(h) / \sum_{j=1}^{m} \omega_{j}^{0}(h), \ldots, \omega_{m}^{0}(h) / \sum_{j=1}^{m} \omega_{j}^{0}(h)\right)^{T}
$$

Once the optimal weights and the IVIFPIS are obtained from (4.5) and (4.6), the distance between each alternative and the IVIFPIS can be calculated for each DM $d_{p}$ as $332 S_{i}^{p}$ based on (3.8), from which a ranking of all alternatives can be derived accordingly 333 for $\mathrm{DM} d_{p}(p=1,2, \ldots, q)$.

334 Linear program (4.4) possesses a fine property that makes it convenient to apply the 
proposed method.

336 Theorem 4.1 If $h$ in the first constraint of the linear program (4.4) is changed to a different positive number, the optimal IVIFPIS determined by (4.5) and the normalized weight vector calculated by (4.6) remain optimal.

Proof. Let $\hat{h}>0$ and $\hat{h} \neq h$. Multiplying the objective function and both sides of the 340 constraints in (4.4) by $\frac{\hat{h}}{h}$ yields the following linear program:

$$
\begin{aligned}
& \min \left\{\sum_{p=1}^{q} \sum_{(k, t) \in \Omega^{p}} \xi_{k t}^{p} \frac{\hat{h}}{h}\right\} \\
& \text { s.t. } \sum_{j=1}^{m} \omega_{j} \frac{\hat{h}}{h} F_{j}+\sum_{j=1}^{m} \hat{a}_{j} \frac{\hat{h}}{h} C_{j}+\sum_{j=1}^{m} \hat{b}_{j} \frac{\hat{h}}{h} G_{j}+\sum_{j=1}^{m} \hat{c}_{j} \frac{\hat{h}}{h} H_{j}+\sum_{j=1}^{m} \hat{d}_{j} \frac{\hat{h}}{h} T_{j} \geq h \frac{\hat{h}}{h}=\hat{h} \\
& \sum_{j=1}^{m} \omega_{j} \frac{\hat{h}}{h} F_{t j k}^{p}+\sum_{j=1}^{m} \hat{a}_{j} \frac{\hat{h}}{h} C_{t j k}^{p}+\sum_{j=1}^{m} \hat{b}_{j} \frac{\hat{h}}{h} G_{t j k}^{p}+\sum_{j=1}^{m} \hat{c}_{j} \frac{\hat{h}}{h} H_{t j k}^{p}+\sum_{j=1}^{m} \hat{d}_{j} \frac{\hat{h}}{h} T_{t j k}^{p}+\xi_{k t}^{p} \frac{\hat{h}}{h} \geq 0 \\
& \left((k, t) \in \Omega^{p} ; p=1,2, \ldots, q\right) \\
& \xi_{k t}^{p} \frac{\hat{h}}{h} \geq 0 \quad\left((k, t) \in \Omega^{p} ; p=1,2, \ldots, q\right) \\
& \hat{b}_{j} \frac{\hat{h}}{h}+\hat{d}_{j} \frac{\hat{h}}{h} \leq \omega_{j} \frac{\hat{h}}{h}, \hat{a}_{j} \frac{\hat{h}}{h} \leq \hat{b}_{j} \frac{\hat{h}}{h}, \hat{c}_{j} \frac{\hat{h}}{h} \leq \hat{d}_{j} \frac{\hat{h}}{h}(j=1,2, \ldots, m) \\
& \hat{a}_{j} \frac{\hat{h}}{h} \geq 0, \hat{b}_{j} \frac{\hat{h}}{h} \geq 0, \hat{c}_{j} \frac{\hat{h}}{h} \geq 0, \hat{d}_{j} \frac{\hat{h}}{h} \geq 0 \quad(j=1,2, \ldots, m) \\
& \omega_{j} \frac{\hat{h}}{h} \geq 0 \quad(j=1,2, \ldots, m) .
\end{aligned}
$$

342 Let $\xi_{k t}^{\prime p} \triangleq \xi_{k t}^{p} \frac{\hat{h}}{h}, \omega_{j}^{\prime} \triangleq \omega_{j} \frac{\hat{h}}{h}, \hat{a}_{j}^{\prime} \triangleq \hat{a}_{j} \frac{\hat{h}}{h}, \hat{b}_{j}^{\prime} \triangleq \hat{b}_{j} \frac{\hat{h}}{h}, \hat{c}_{j}^{\prime} \triangleq \hat{c}_{j} \frac{\hat{h}}{h}$, and $\hat{d}_{j}^{\prime} \triangleq \hat{d}_{j} \frac{\hat{h}}{h}$, it is apparent that

343 the aforesaid linear program is identical to (4.4) except for the relabeled decision

344 variables and the right-hand value of the first constraint. Then $\omega_{j}^{0}(\hat{h})=\frac{\hat{h}}{h} \omega_{j}^{0}(h)$,

$345 \quad \hat{a}_{j}^{\prime 0}(\hat{h})=\frac{\hat{h}}{h} \hat{a}_{j}^{0}(h), \hat{b}_{j}^{\prime 0}(\hat{h})=\frac{\hat{h}}{h} \hat{b}_{j}^{0}(h), \hat{c}_{j}^{\prime 0}(\hat{h})=\frac{\hat{h}}{h} \hat{c}_{j}^{0}(h)$, and $\hat{d}_{j}^{\prime 0}(\hat{h})=\frac{\hat{h}}{h} \hat{d}_{j}^{0}(h)(j=1,2, \ldots, m)$.

346 Therefore, we have

$$
\tilde{r}_{j}^{* * 0}(\hat{h})=\left(\left[\frac{\hat{a}_{j}^{0}(\hat{h})}{\omega_{j}^{0}(\hat{h})}, \frac{\hat{b}_{j}^{0}(\hat{h})}{\omega_{j}^{0}(\hat{h})}\right],\left[\frac{\hat{c}_{j}^{0}(\hat{h})}{\omega_{j}^{0}(\hat{h})}, \frac{\hat{d}_{j}^{0}(\hat{h})}{\omega_{j}^{0}(\hat{h})}\right]\right)=\left(\left[\frac{\hat{a}_{j}^{0}(h)}{\omega_{j}^{0}(h)}, \frac{\hat{b}_{j}^{0}(h)}{\omega_{j}^{0}(h)}\right],\left[\frac{\hat{c}_{j}^{0}(h)}{\omega_{j}^{0}(h)}, \frac{\hat{d}_{j}^{0}(h)}{\omega_{j}^{0}(h)}\right]\right)=\tilde{r}_{j}^{* 0}(h)
$$


348 and $\omega_{j}^{0}(\hat{h}) / \sum_{j=1}^{m} \omega_{j}^{0}(\hat{h})=\left(\frac{\hat{h}}{h} \omega_{j}^{0}(h)\right) / \sum_{j=1}^{m} \frac{\hat{h}}{h} \omega_{j}^{0}(h)=\omega_{j}^{0}(h) / \sum_{j=1}^{m} \omega_{j}^{0}(h)(j=1,2, \ldots, m)$.

349 Theorem 4.1 indicates that the parameter value $h$ in the linear program (4.4) is 350 irrelevant in determining the optimal IVIFPIS and normalized weight vector. The 351 implication is that an analyst can select any positive $h$ value to calibrate the model.

352 Based on the aforesaid analyses, we are now in a position to formulate an interval353 valued intuitionistic fuzzy approach to MAGDM as described in the following steps.

354 Step 1. Convert linguistic assessments on alternative $x_{i} \in X$ to appropriate IVIFNs for 355 qualitative attributes $a_{j} \in A_{1}$.

356 Step 2. Calculate corresponding IVIFNs for numerical assessments on alternative $357 x_{i} \in X$ for quantitative attributes $a_{j} \in A_{2}$ as per (3.1) and (3.4).

358 Step 3. Construct the IVIFN decision matrix $\tilde{R}^{p}=\left(\tilde{r}_{i j}^{p}\right)_{n \times m}=\left(\left(\left[a_{i j}^{p}, b_{i j}^{p}\right],\left[c_{i j}^{p}, d_{i j}^{p}\right]\right)\right)_{n \times m}$ 359 for $\mathrm{DM}_{p}(p=1,2, \ldots, q)$.

360 Step 4. Establish the linear programming model (4.4) based on the incomplete pairwise 361 comparison preference relations furnished by the DMs.

362 Step 5. Obtain the optimal values $\omega_{j}^{0}(h), \hat{a}_{j}^{0}(h), \hat{b}_{j}^{0}(h), \hat{c}_{j}^{0}(h)$ and $\hat{d}_{j}^{0}(h)(j=1,2, \ldots$, $363 m$ ) by solving (4.4) with any given parameter $h>0$.

364 Step 6. Calculate the optimal normalized weight vector as per (4.6).

365 Step 7. Determine the optimal IVIFPIS $x^{* 0}(h)=\left(\tilde{r}_{1}^{* 0}(h), \tilde{r}_{2}^{* 0}(h), \ldots, \tilde{r}_{m}^{* 0}(h)\right)^{T}$ as per (4.5).

366 Step 8. Compute the weighted average of squared Euclidean distances $S_{i}^{p}$ between 367 alternatives $x_{i}$ and the IVIFPIS $x^{* 0}(h)$ as per (3.8) $(i=1,2, \ldots, n, p=1,2, \ldots, q)$.

368 Step 9. Rank all alternatives for $\mathrm{DM} d_{p}(p=1,2, \ldots, q)$ according to an increasing 369 order of their distances $S_{i}^{p}(i=1,2, \ldots, n)$.

370 Step 10. Rank all alternatives for the group using the Borda function (Hwang \& Yoon, 371 1981) and the best alternative is the one with the smallest Borda scores.

\section{An illustrative example}


This section presents an MAGDM problem about recommending undergraduate students for graduate admission to demonstrate how to apply the proposed approach.

375 Without loss of generality, assume that there are three committee members (i.e., DMs) $376 d_{1}, d_{2}$, and $d_{3}$, and four students $x_{1}, x_{2}, x_{3}$, and $x_{4}$ as the finalists after preliminary 377 screening. All DMs agree to evaluate these candidates against four attributes, academic records $\left(a_{1}\right)$, college English test Band 6 score $\left(a_{2}\right)$, teamwork skills $\left(a_{3}\right)$, and research potentials $\left(a_{4}\right) . a_{1}$ is assessed based the cumulative grade point average (GPA), and $a_{2}$ is assessed out of 710 points with a minimum qualifying level of 425 points. $a_{1}$ and $a_{2}$ are both benefit quantitative attributes. $a_{3}$ and $a_{4}$ can be well characterized as qualitative attributes and their ratings can be easily expressed as linguistic variables. This example assumes that the group has agreed to assess qualitative attributes on five linguistic terms as given in Table 1, which also provides a conversion table between linguistic terms and IVIFNs. Assume that the three committee members have furnished their assessments of the four candidates on the four attributes as shown in Table 2.

Table 2. Raw decision data furnished by the DMs

\begin{tabular}{||c|c|c|c|c|c||}
\hline \multirow{2}{*}{ Experts } & \multirow{2}{*}{ Students } & \multicolumn{4}{|c||}{ Attributes } \\
\cline { 2 - 6 } & & $a_{1}$ & $a_{2}$ & $a_{3}$ & $a_{4}$ \\
\hline \multirow{4}{*}{$d_{1}$} & $x_{1}$ & 88 & 550 & $\mathrm{~F}$ & $\mathrm{VG}$ \\
\cline { 2 - 6 } & $x_{2}$ & 96 & 520 & $\mathrm{P}$ & $\mathrm{F}$ \\
\cline { 2 - 6 } & $x_{3}$ & 92 & 580 & $\mathrm{G}$ & $\mathrm{G}$ \\
\cline { 2 - 6 } & $x_{4}$ & 90 & 500 & $\mathrm{~F}$ & $\mathrm{~F}$ \\
\hline \multirow{6}{*}{$d_{2}$} & $x_{1}$ & 88 & 550 & $\mathrm{G}$ & $\mathrm{G}$ \\
\cline { 2 - 6 } & $x_{2}$ & 96 & 520 & $\mathrm{P}$ & $\mathrm{F}$ \\
\cline { 2 - 6 } & $x_{3}$ & 92 & 580 & $\mathrm{~F}$ & $\mathrm{VG}$ \\
\cline { 2 - 6 } & $x_{4}$ & 90 & 500 & $\mathrm{~F}$ & $\mathrm{~F}$ \\
\hline$d_{3}$ & $x_{1}$ & 88 & 550 & $\mathrm{~F}$ & $\mathrm{VG}$ \\
\cline { 2 - 6 } & $x_{2}$ & 96 & 520 & $\mathrm{P}$ & $\mathrm{F}$ \\
\cline { 2 - 6 } & $x_{3}$ & 92 & 580 & $\mathrm{~F}$ & $\mathrm{~F}$ \\
\cline { 2 - 6 } & $x_{4}$ & 90 & 500 & $\mathrm{G}$ & $\mathrm{F}$ \\
\hline \hline
\end{tabular}

Assume further that the DMs provide their incomplete pariwise comparison preference relations on the four candidates as follows:

$403(p=1,2,3)$. For this particular example, the assessment values on the two quantitative 
attributes are common for the three DMs given that they are simply taken from the four candidates' historical records. However, it is worth noting that the proposed model in this paper is able to handle the case where each DM provides different assessments for quantitative attributes.

For the same quantitative assessment, it is understandable that different DMs may have different opinions on how well it satisfies a particular attribute. For instance, what percentage grade can be converted to a letter grade of $\mathrm{A}$ ? The answer to this question depends on what grade conversion scale is adopted by an instructor. Therefore, it is sensible that each DM may have different degrees of satisfaction and non-satisfaction for

413 the same quantitative assessment. It is assumed that $\mathrm{DM} d_{p}, p=1,2,3$, provide their 414 degrees of satisfaction for $f_{1 p}^{\max }=96$ as $\bar{\beta}_{1}^{1}=\left[\beta_{1}^{1 l}, \beta_{1}^{1 u}\right]=[0.90,0.95], \bar{\beta}_{1}^{2}=\left[\beta_{1}^{2 l}, \beta_{1}^{2 u}\right]=$ $415[0.85,0.90]$, and $\bar{\beta}_{1}^{3}=\left[\beta_{1}^{3 l}, \beta_{1}^{3 u}\right]=[0.86,0.92]$; degrees of non-satisfaction as $\left[\hat{c}_{1}^{21}, \hat{d}_{1}^{21}\right]$ $416=[0.02,0.03],\left[\hat{c}_{1}^{22}, \hat{d}_{1}^{22}\right]=[0.05,0.08]$, and $\left[\hat{c}_{1}^{23}, \hat{d}_{1}^{23}\right]=[0.05,0.07]$, respectively.

417 Similarly, assume that $\mathrm{DM} d_{p}, p=1,2,3$, furnish their degree of satisfaction for $418 f_{2 p}^{\max }=580$ as $\bar{\beta}_{2}^{1}=\left[\beta_{2}^{1 l}, \beta_{2}^{1 u}\right]=[0.88,0.92], \bar{\beta}_{2}^{2}=\left[\beta_{2}^{2 l}, \beta_{2}^{2 u}\right]=[0.9,0.92]$, and $\bar{\beta}_{2}^{3}=$ $\left[\beta_{2}^{3 l}, \beta_{2}^{3 u}\right]=[0.85,0.90]$, and $\left[\hat{c}_{2}^{21}, \hat{d}_{2}^{21}\right]=[0.03,0.06],\left[\hat{c}_{2}^{22}, \hat{d}_{2}^{22}\right]=[0.03,0.05]$, and $\left[\hat{c}_{2}^{23}, \hat{d}_{2}^{23}\right]=[0.05,0.07]$, respectively.

Based on (3.1), one can derive each DM's degrees of satisfaction for the four candidates against the two quantitative attributes as the first intervals in every cell of the 423 first two columns in Tables 3, 4, and 5.

424 By using (3.3), one can determine: $\bar{\gamma}_{1}^{1}=[0.40,0.60], \quad \bar{\gamma}_{1}^{2}=[0.50,0.80], \quad \bar{\gamma}_{1}^{3}=$ $425[0.625,0.875], \bar{\gamma}_{2}^{1}=[0.375,0.75], \quad \bar{\gamma}_{2}^{2}=[0.375,0.625], \bar{\gamma}_{2}^{3}=[0.50,0.70]$. According to 426 (3.4), each DM's degrees of nonsatisfaction for all candidates for the two quantitative 427 attributes are derived as the second intervals in every cell of the first two columns in 428 Tables 3, 4, and 5.

429 As per Table 1, the linguistic assessments on the two qualitative attributes can be 430 converted to interval-valued intuitionistic fuzzy data. The result is shown in the last two 431 columns of the decision matrices for $\operatorname{DM} d_{p}(p=1,2,3)$ in Tables 3, 4, and 5: 
432 Table 3. Interval-valued intuitionistic fuzzy decision matrix for DM $d_{1} \tilde{R}^{1}$

433

\begin{tabular}{ccccc}
\hline & $a_{1}$ & $a_{2}$ & $a_{3}$ & $a_{4}$ \\
\hline$x_{1}$ & $([0.0000,0.0000],[0.4000,0.6000])$ & $([0.5500,0.5750],[0.1594,0.3188])$ & $([0.50,0.55],[0.40,0.45])$ & $([0.90,0.95],[0.02,0.05])$ \\
$x_{2}$ & $([0.9000,0.9500],[0.0200,0.0300])$ & $([0.2200,0.2300],[0.2888,0.5775])$ & $([0.20,0.25],[0.70,0.75])$ & $([0.50,0.55],[0.40,0.45])$ \\
$x_{3}$ & $([0.4500,0.4750],[0.2100,0.3150])$ & $([0.8800,0.9200],[0.0300,0.0600])$ & $([0.70,0.75],[0.20,0.25])$ & $([0.70,0.75],[0.20,0.25])$ \\
$x_{4}$ & $([0.2250,0.2375],[0.3050,0.4575])$ & $([0.0000,0.0000],[0.3750,0.7500])$ & $([0.50,0.55],[0.40,0.45])$ & $([0.50,0.55],[0.40,0.45])$ \\
\hline
\end{tabular}

435

436 Table 4. Interval-valued intuitionistic fuzzy decision matrix for $\mathrm{DM} d_{2} \tilde{R}^{2}$

\begin{tabular}{ccccc}
\hline & $a_{1}$ & $a_{2}$ & $a_{3}$ & $a_{4}$ \\
\hline$x_{1}$ & $([0.0000,0.0000],[0.5000,0.8000])$ & $([0.5625,0.5750],[0.1594,0.2656])$ & $([0.70,0.75],[0.20,0.25])$ & $([0.70,0.75],[0.20,0.25]))$ \\
$x_{2}$ & $([0.8500,0.9000],[0.0500,0.0800])$ & $([0.2250,0.2300],[0.2888,0.4813])$ & $([0.20,0.25],[0.70,0.75])$ & $([0.50,0.55],[0.40,0.45])$ \\
$x_{3}$ & $([0.4250,0.4500],[0.2750,0.4400])$ & $([0.9000,0.9200],[0.0300,0.0500])$ & $([0.50,0.55],[0.40,0.45])$ & $([0.90,0.95],[0.02,0.05])$ \\
$x_{4}$ & $([0.2125,0.2250],[0.3875,0.6200])$ & $([0.0000,0.0000],[0.3750,0.6250])$ & $([0.50,0.55],[0.40,0.45])$ & $([0.50,0.55],[0.40,0.45])$ \\
\hline
\end{tabular}

440 Table 5. Interval-valued intuitionistic fuzzy decision matrix for DM $d_{3} \tilde{R}^{3}$

$x_{1} \quad([0.0000,0.0000],[0.6250,0.8750]) \quad([0.5313,0.5625],[0.2188,0.3063]) \quad([0.50,0.55],[0.40,0.45]) \quad([0.90,0.95],[0.02,0.05])$

$\begin{array}{lllll}x_{2} & ([0.8600,0.9200],[0.0500,0.0700]) & ([0.2125,0.2250],[0.3875,0.5425]) & ([0.20,0.25],[0.70,0.75]) & ([0.50,0.55],[0.40,0.45])\end{array}$

$\begin{array}{lllll}x_{3} & ([0.4300,0.4600],[0.3375,0.4725]) & ([0.8500,0.9000],[0.0500,0.0700]) & ([0.50,0.55],[0.40,0.45]) & ([0.50,0.55],[0.40,0.45])\end{array}$

$x_{4} \quad([0.2150,0.2300],[0.4813,0.6783]) \quad([0.0000,0.0000],[0.5000,0.7000]) \quad([0.70,0.75],[0.20,0.25]) \quad([0.50,0.55],[0.40,0.45])$

444 It can be seen from the interval-valued intuitionistic fuzzy decision matrix $\tilde{R}^{1}$ that

445 DM $d_{1}$ 's degrees of satisfaction and non-satisfaction for $x_{2}$ on $a_{1}$ are computed as

$446[0.9000,0.9500]$ and $[0.0200,0.0300]$ rather than $[1,1]$ and $[0,0]$ although $x_{2}$ reaches the

447 maximum $f_{11}^{\max }=96$. This conversion process presumably reflects that DM $d_{1}$ is not

448 completely satisfied with candidate $a_{1}$ 's cumulative GPA $f_{11}^{\max }=96$ although this student

449 achieves the highest GPA among the four candidates. Similarly, $\tilde{r}_{31}^{1}$ indicates that DM

$450 d_{1}$ 's degrees of satisfaction and non-satisfaction for $x_{3}$ on $a_{1}$ are [0.45, 0.475] and [0.21,

451 0.315], respectively. This converted IVIFN assessment points to a hesitancy degree of

$452[0.21,0.34]$ for $\mathrm{DM} d_{1}$. 
As per Theorem 4.1, the parameter $h$ in (4.4) can be arbitrarily selected without 454 affecting the optimal normalized weights and IVIFPIS. By setting $h=1$, solving model 455 (4.4) yields the following optimal solution:

$$
\left(\omega_{1}^{0}, \omega_{2}^{0}, \omega_{3}^{0}, \omega_{4}^{0}\right)^{T}=(701.5739,1030.2918,394.9273,485.3135)^{T},
$$

$$
\left(\hat{a}_{1}^{0}, \hat{a}_{2}^{0}, \hat{a}_{3}^{0}, \hat{a}_{4}^{0}\right)^{T}=(290.1888,343.5678,129.3340,166.3520)^{T},
$$

$$
S_{1}^{1}=0.120194, S_{2}^{1}=0.120192, S_{3}^{1}=0.120181, S_{4}^{1}=0.120159 \text {, }
$$

$$
(0.2686,0.3944,0.1512,0.1858)^{T} \text {. }
$$

By using (4.6), one can obtain the optimal normalized weight vector as

$$
\text { As per (4.5), the optimal IVIFPIS is determined as }
$$

$$
\begin{aligned}
x^{*_{0}}= & (([0.4136,0.5606],[0.0649,0.1489]),([0.3335,0.4802],[0.1622,0.2240]), \\
& ([0.3275,0.5284],[0.1205,0.2795]),([0.3428,0.5516],[0.0981,0.2489]))^{T}
\end{aligned}
$$

According to (3.8), the weighted average of squared Euclidean distances $S_{i}^{p}(i=1$, $2, \ldots, 4, p=1,2,3)$ between $x_{i}$ and the IVIFPIS can be calculated as follows:

$$
\begin{aligned}
& S_{1}^{2}=0.123826, S_{2}^{2}=0.105802, S_{3}^{2}=0.146691, S_{4}^{2}=0.123683, \\
& S_{1}^{3}=0.157639, S_{2}^{3}=0.117237, S_{3}^{3}=0.125221, S_{4}^{3}=0.148978 .
\end{aligned}
$$
$x_{2} \succ_{2} x_{4} \succ_{2} x_{1} \succ_{2} x_{3}$ and $x_{2} \succ_{3} x_{3} \succ_{3} x_{4} \succ_{3} x_{1}$, respectively, where $x_{k} \succ_{p} x_{t}$ indicates that

472 Using the Borda function (Hwang \& Yoon, 1981), Borda scores of the four 473 candidates can be determined as shown in the last column of Table 6.

474 The final group ranking of the four alternatives can thus be obtained as $475 \quad x_{2} \succ x_{4} \succ x_{3} \succ x_{1}$. 
Table 6. Borda scores of the four candidates

\begin{tabular}{||c|c|c|c|c||}
\hline \multirow{2}{*}{ Candidate } & \multicolumn{3}{|c||}{ Decision-maker } & \multirow{2}{*}{ Borda score } \\
\cline { 2 - 5 } & $d_{1}$ & $d_{2}$ & $d_{3}$ & \\
\hline$x_{1}$ & 3 & 2 & 3 & 8 \\
\hline$x_{2}$ & 2 & 0 & 0 & 2 \\
\hline$x_{3}$ & 1 & 3 & 1 & 5 \\
\hline$x_{4}$ & 0 & 1 & 2 & 3 \\
\hline
\end{tabular}

\section{Conclusions}

486 In a typical MAGDM problem, both quantitative and qualitative attributes are often 487 involved and assessed with imprecise data and subjective judgment. This article first 488 proposes mechanisms for converting numerical quantitative assessments and linguistic 489 qualitative values into IVIFN decision data. Based on incomplete pairwise comparison 490 preference relations furnished by the DMs, group consistency and inconsistency indices 491 are introduced. The converted IVIFN decision data and group consistency and 492 inconsistency indices are then employed to establish a linear programming model for 493 determining unified attribute weights and IVIFPIS. An illustrative numerical example is

494 developed to demonstrate how to apply the proposed framework.

495 Current research assumes that qualitative and quantitative attributes are assessed as 496 linguistic terms and numerical values, respectively. Additional research is needed to

497 handle the case when the corresponding assessments are expressed as interval linguistic 498 variables and interval numbers. Moreover, the current linear program (4.4) assumes that 499 each DM has the same influence over the decision process. It is a worthy topic to address 500 the situation that different DMs exert distinct weights on choosing the final alternative.

\section{REFERENCES}

502 Atanassov, K. (1986). Intuitionistic fuzzy sets. Fuzzy Sets and Systems, 20, 87-96.

503 Atanassov, K. \& Gargov, G. (1989). Interval-valued intuitionistic fuzzy sets. Fuzzy Sets and Systems 504 31, 343-349.

505 Bustince, H. \& Burillo, P. (1995). Correlation of interval-valued intuitionistic fuzzy sets. Fuzzy Sets 506 and Systems, 74, 237-244. 
Chen, T.Y., Wang, H.P., \& Lu, Y.Y. (2011). A multicriteria group decision-making approach based on interval-valued intuitionistic fuzzy sets: A comparative perspective. Expert Systems with Applications, 38, 7647-7658.

Deschrijver, G. (2007). Arithmetic operators in interval-valued fuzzy set theory. Information Sciences, $177,2906-2924$.

Deschrijver, G. (2008). A representation of t-norms in interval-valued L-fuzzy set theory. Fuzzy Sets and Systems, 159, 1597-1618.

Deschrijver, G. \& Kerre, E.E. (2007). On the position of intuitionistic fuzzy set theory in the framework of theories modelling imprecision. Information Sciences, 177, 1860 - 1866.

Hong, D.H. (1998). A note on correlation of interval-valued intuitionistic fuzzy sets. Fuzzy Sets and Systems, 95, 113-117.

Hung, W.L. \& Wu, J.W. (2002). Correlation of intuitionistic fuzzy sets by centroid method. Information Sciences, 144, 219 - 225.

Hwang, C. L. \& Yoon, K. (1981). Multiple Attribute Decision Making: Methods and Applications. Springer, Berlin, Heideberg, New York, 1981.

Li, D. F. (2005). Multiattribute decision making models and methods using intuitionistic fuzzy sets. Journal of Computer and System Sciences, 70, 73-85.

Li, D.F. (2010). Linear programming method for MADM with interval-valued intuitionistic fuzzy sets. Expert Systems with Applications, 37, 5939-5945.

Li, D.F. (2010). TOPSIS-based nonlinear-programming methodology for multiattribute decision making with interval-valued intuitionistic fuzzy sets. IEEE Transactions on Fuzzy Systems, 18, 299-311

Li, D.F. (2011). Closeness coefficient based nonlinear programming method for interval-valued intuitionistic fuzzy multiattribute decision making with incomplete preference information. Applied Soft Computing, 11, 3402-3418.

Li, D.F., Chen, G..H., \& Huang, Z.G. (2010). Linear programming method for multiattribute group decision making using IF sets. Information Sciences, 180, 1591-1609.

Li, D.F. \& Yang, J.B. (2004). Fuzzy linear programming technique for multiattribute group decision making in fuzzy environments. Information Sciences, 158, 263-275.

Li, K.W. \& Wang, Z. (2010). Notes on "Multicriteria Fuzzy Decision-making Method Based on a Novel Accuracy Function under Interval-valued Intuitionistic Fuzzy Environment", Journal of Systems Science and Systems Engineering, 19, 504-508.

Park, J.H., Park, I.Y., Kwun, Y.C., \& Tan, X. (2011). Extension of the TOPSIS method for decision making problems under interval-valued intuitionistic fuzzy environment. Applied Mathematical Modelling, 35, 2544-2556. 
542 Park, D.G., Kwun, Y.C., Park, J.H., \& Park, I.Y. (2009). Correlation coefficient of interval-valued 543 intuitionistic fuzzy sets and its application to multiple attribute group decision making problems. 544 Mathematical and Computer Modelling, 50, 1279-1293.

545 Srinivasan, V. \& Shocker, A.D. (1973). Linear programming techniques for multidimensional analysis 546 of preference. Psychometrica, 38, 337-342.

547 Wang, Z., Li, K.W., \& Wang, W. (2009). An approach to multiattribute decision making with 548 interval-valued intuitionistic fuzzy assessments and incomplete weights. Information Sciences, 179, 3026-3040.

Wang, Z., Li, K.W., \& Xu, J. (2011). A mathematical programming approach to multi-attribute

Wang, Z., Wang, L., \& Li, K.W. (2011). A linear programming method for interval-valued intuitionistic fuzzy multiattribute group decision making, In Proceedings of the 2011 Chinese Control and Decision Conference, 3833-3838, Mianyang, China.

Wei, C. P., Wang, P., Zhang, Y. Z. (2011). Entropy, similarity measure of interval-valued intuitionistic fuzzy sets and their applications. Information Sciences, 181, 4273-4286.

Wei, G. (2010). Some induced geometric aggregation operators with intuitionistic fuzzy information and their application to group decision making. Applied Soft Computing , 10, 423-431.

Wei, G. (2011). Gray relational analysis method for intuitionistic fuzzy multiple attribute decision making. Expert Systems with Applications, 38, 11671-11677.

Xu, K., Zhou, J., Gu, R. \& Qin, H. (2011). Approach for aggregating interval-valued intuitionistic fuzzy information and its application to reservoir operation. Expert Systems with Applications, 38, 9032-9035.

$\mathrm{Xu}, \mathrm{Z}$. (2007). Methods for aggregating interval-valued intuitionistic fuzzy information and their application to decision making. Control and Decision, 22, 215-219 (in Chinese).

$\mathrm{Xu}, \mathrm{Z}$. (2010). A method based on distance measure for interval-valued intuitionistic fuzzy group decision making. Information Sciences, 180, 181-190.

Xu, Z. \& Cai, X. (2010). Recent advances in intuitionistic fuzzy information aggregation. Fuzzy Optimization and Decision Making, 9, 359-381.

Xu, Z. \& Chen, J. (2008). An overview of distance and similarity measures of intuitionistic fuzzy sets. International Journal of Uncertainty, Fuzziness and Knowledge-Based Systems, 16, 529-555.

$\mathrm{Xu}$, Z. \& Yager, R. R. (2008). Dynamic intuitionistic fuzzy multi-attribute making. International Journal of Approximate Reasoning, 48, 246-262.

Ye, F. (2010). An extended TOPSIS method with interval-valued intuitionistic fuzzy numbers for virtual enterprise partner selection. Expert Systems with Applications, 37, 7050-7055. 
577 Ye, J. (2011). Fuzzy cross entropy of interval-valued intuitionistic fuzzy sets and its optimal decisionmaking method based on the weights of alternatives. Expert Systems with Applications, 38, 61796183.

580 Yue, Z. (2011). An approach to aggregating interval numbers into interval-valued intuitionistic fuzzy information for group decision making. Expert Systems with Applications, 38, 6333-6338.

582 Zadeh, L.A. (1965). Fuzzy sets. Information and Control, 8 , 338-356. 